\title{
CARACTERIZACIÓN Y TEMPORALIDAD DE ENEMIGOS NATURALES DE Opsiphanes cassina EN PALMA ACEITERA, ESTADO MONAGAS, VENEZUELA
}

\section{CHARACTERIZATION AND TEMPORALITY OF NATURAL ENEMIES OF Opsiphanes cassina IN OIL PALM, MONAGAS STATE, VENEZUELA}

\author{
Gladys Rodríguez Gonzalez ${ }^{1}$, Ramón Silva-Acuña ${ }^{2}$, Rafael Cásares Moizant ${ }^{3}$, Asdrúbal Díaz Quintana ${ }^{1}$, Renny Barrios \\ Maestre $^{1}$ \\ ${ }^{1}$ Instituto Nacional de Investigaciones Agrícolas, Centro de Investigaciones Agrícolas del estado Monagas. Apartado Postal \\ 184. Z.P. 6201. Maturín, Monagas, Venezuela \\ ${ }^{2}$ Universidad de Oriente, Núcleo de Monagas, Escuela de Ingeniería Agronómica, Postgrado de Agricultura Tropical, \\ Maturín, Venezuela \\ ${ }^{3}$ Instituto de Zoología Agrícola, Facultad de Agronomía, Universidad Central de Venezuela, Apartado Postal 4579. \\ Z.P.2101-A. Maracay, Aragua, Venezuela. \\ E-mai: rennybarrios@gmail.com
}

\section{Información del articulo}

Tipo de artículo: Artículo original

Recibido:

08/02/2021

Aceptado:

27/05/2021

Licencia:

CC BY-NC-SA 4.0

Revista

ESPAMCIENCIA

12(1):17-24

DOI:

https://doi.org/10.51

260/revista_espamci

encia.v12i1.261

\section{Resumen}

La investigación se condujo con el objetivo de realizar la caracterización y temporalidad de los enemigos naturales asociados con Opsiphanes cassina en una plantación de palma aceitera, ubicada en el estado Monagas, Finca "El Águila" Venezuela. Se efectuaron evaluaciones a intervalos semanales, durante el ciclo agosto 2015/agosto 2016, seccionando la hoja número 25 de dos plantas.ha ${ }^{-1}$ en un lote de 10 ha. Se cuantificó el número de huevos, larvas diferentes instares y pupas, así como también, de sus enemigos naturales y las especies vegetales que les ofrecen alimento y refugio dentro del área experimental. Los agentes de control biológico identificados correspondieron a Telenomus sp. (Scelionidae), Anastatus sp. (Eupelmidae) y un eulófido como parasitoides de huevos; Cotesia sp. (Braconidae) de larvas del V instar y Conura maculata $\mathrm{F}$ (Chalcididae) de pupas. Cotesia sp., fue el entomófago más relevante en la regulación de las poblaciones del insecto. La presencia de especies como Croton trinitatis Mills y Solanum hirtum L favorecen el fortalecimiento del control biológico natural de O. cassina.

Palabras clave: plantas arvenses, Elaeis guinensis, gusano de las palmeras, control biológico, parasitoides

\begin{abstract}
The research was conducted with the objective of performing the characterization and temporality of the natural enemies associated with Opsiphanes cassina in an oil palm plantation, located in Monagas state, Finca "El Águila" Venezuela. Evaluations were carried out at weekly intervals, during the August 2015/August 2016 cycle, sectioning leaf number 25 from two plants ha- 1 in a 10 ha lot. The number of eggs, different instars larvae and pupa, as well as their natural enemies and the plant species that offer them food and shelter within the experimental area, were quantified. The biological control agents identified corresponded to Telenomus sp. (Scelionidae), Anastatus sp. (Eupelmidae) and a eulophyid as egg parasitoids; Cotesia sp. (Braconidae) from larvae of V instar and Conura maculata F (Chalcididae) from pupa. Cotesia $s p$., was the most relevant entomophagus in the regulation of insect populations. The presence of species such as Croton trinitatis Mills and Solanum hirtum $L$ favor the strengthening of the natural biological control of O. cassina.
\end{abstract}

Key words: weed plants, Elaeis guinensis, palm worm, biological control, parasitoids. 


\section{INTRODUCCIÓN}

La palma aceitera (Elaeis guineensis Jacq) dentro de las oleaginosas, es un cultivo de relevante importancia económica, sus productos, están dirigidos tanto a la producción de alimentos como para la industria oleoquimica; además, su cultivo genera impacto social al crear empleos y también beneficia lo ambiental, protegiéndolo. USDA (2020) proyecta para 2020/2021 una producción mundial de aceite de palma de 74,60 millones de toneladas métricas (TM), siendo la del ciclo 2019/2020 de 72,27 millones de TM.

En Venezuela, Díaz y Barrios (2007) consideran al cultivo de palma aceitera como el proyecto que generó el mayor desarrollo agrícola e industrial. En el país, se consumen $560000 \mathrm{TM}^{2}$ año ${ }^{-1}$ de aceites y grasas vegetales, y solo se producen $120000 \mathrm{TM}$; por tanto, existe un déficit de 79\%, que obliga a mantener las importaciones.

O. cassina, es la especie de insecto defoliador más dañino, capaz de causar severas defoliaciones en plantas adultas de palma aceitera y cocotero y esporádicamente en plantas jóvenes (Rodríguez et al., 2012a; Oliveira et al., 2018), existen registros de más de 600 larvas.hojas ${ }^{-1}$ (Chinchilla, 2003; Aldana y Aldana, 2017). Para realizar el control de larvas defoliadoras en plantaciones de palma aceitera se requiere realizar censos regulares, así como también, la aplicación de productos selectivos para reducir las poblaciones a niveles del equilibrio natural. Ese equilibrio, es mantenido principalmente por chinches depredadoras, parasitoides y entomopatógenos como hongos y virus. En ausencia del control natural, las larvas son capaces de incrementar rápidamente sus poblaciones y causar severos daños (Chung et al., 1996).

Las cuantificaciones poblacionales de $O$. cassina, se efectúan normalmente mediante recuentos semanales en la hoja 17 o 25 en una o dos palmas por ha (Rodríguez et al., 2011; Oliveira et al., 2018). El muestreo oportuno de las diferentes etapas de desarrollo del insecto plaga (Rodríguez et al., 2012b) y sus enemigos naturales, es la base de un programa de Manejo Integrado de Plagas (MIP) (Díaz-Castañeda, et al., 2020) ya que permite definir la ubicación e importancia del problema cuando todavía se limita a unas pocas plantas y tener mayor dominio para establecer las medidas más razonables para enfrentarlo (Chinchilla, 2003; Calvache, 2016).

Para la región del sur del Lago de Maracaibo, estado Zulia (Escalante y Rosales, 2007) señalan los insectos que causan parasitismo a los estados inmaduros de O. cassina y los dividen en $60 \%$ para la fase de pupa, $30 \%$ en larva y $10 \%$ en la fase de huevo, descritos de la siguiente manera: un parasitoide de huevos Telenomus sp. (Hymenoptera: Scelionidae); tres de larvas, Alcaeorhynchus grandis (Hemiptera: Pentatomidae), Aleiodes sp y Cotesia sp. (Hymenoptera: Braconidae) y cinco parasitoides de pupas,
Brachymeria sp (Hymenoptera: Chalcididae); Sarcodexia innata (Diptera: Sarcophagidae); Conura sp (Hymenoptera: Chalcididae); Tetrastichus sp (Hymenoptera: Eulophidae), y Rhysipolis sp (Hymenoptera: Braconidae); también relataron al género Beauveria, como hongo entomopatógeno de insectos defoliadores en palma aceitera.

De manera similar, para los parasitoides de huevos de $O$. cassina, Calvache (2016); Barriga (2016) y Pastrana et al. (2019) describen que son parasitados por Telenomus sp. (Scelionidae) y Oencyrtus sp. (Encyrtidae) mientras que, las larvas tienen como parasitoides a Apanteles sp. y Horismenus sp. (Braconidae), Casinaria sp. (Icheneumonidae), Conura maculata, Conura sp. (Chalcididae), Brachymeria spp, Cotesia sp. (Braconidae); además, de ser depredadas por los chinches pentatómidos, Mormidea ypsilon F, Podisus sp. y Alcaeorrhynchus grandis F (Mexzón y Chinchilla, 1996; Barriga, 2016 y Pastrana et al., 2019); se agrega, que las larvas son afectadas por virus tipos densovirus (Morales $e t$ al., 2017) y el de la poliedrosis nuclear y finalmente, Barriga (2016), señala al entomopatógeno Beauveria bassiana y al reduvido Arilus sp.

Para la pupa, se han señalados varios tachínidos y el chalcidido Spilochalcis nigrifronsy, siendo afectadas por el hongo Beauveria sp y por una bacteria no identificada (Mexzón y Chinchilla, 1996). Recientemente, Barriga (2016) distingue los parasitoides Sarcodesia sp. (Diptera; Sarcophagidae) y Spilochalcis sp. (Himenoptera: Chalcididae); mientras que Pastrana et al. (2019) incluyen a Conura maculata, Conura sp y Brachymeria sp.

Paralelamente con el cultivo, el conocimiento sobre la vegetación asociada, es otro elemento a considerar del ambiente. Muchas plantas son visitadas por parasitoides y depredadores, ellas les ofrecen alimento, refugio y posiblemente, ayudan al desarrollo de patógenos que afectan sus poblaciones. Al menos 75 especies de plantas pertenecientes a varias familias (Leguminosae, Euphorbiacea, Acanthaceae, Compositae, Malvaceae, etc.) han sido consideradas importantes en mantener la diversidad de la entomofauna en plantaciones comerciales de palma aceitera (Mexzón y Chinchilla, 1996), siendo Croton trinitatis (Millsp) la de mejores características para el establecimiento y mantenimiento de parasitoides de insectos defoliadores (Aldana et al., 2010).

En Venezuela, y particularmente en el estado Monagas, se conoce muy poco de las interrelaciones de Opsiphanes cassina con sus biorreguladores y el ambiente para ayudar al mantenimiento del balance del agroecosistema de palma aceitera. Por esta razón, se realizó la presente investigación, con la finalidad de caracterizar y conocer la temporalidad de los controladores biológicos; así como también, realizar observaciones sobre la vegetación 
benéfica en el fortalecimiento del control biológico natural.

\section{MATERIALES Y METODOS}

El ensayo se condujo en la Finca "El Águila", durante el ciclo agosto 2015/agosto 2016, en el lote A2 sur-Vía 3, de 10 ha de palma aceitera del material genético Deli x Avros. El lote asignado para la experimentación abarcó 60 hileras de plantas, las cuales se enumeraron de cinco en cinco, identificándose 11. En cada una de estas hileras se marcaron las plantas con una cinta plástica color amarillo tráfico desde la palma dos hasta la cinco y desde la 14 hasta 17 , creándose 22 estaciones de muestreo, de acuerdo con la metodología desarrollada por Rodríguez et al. (2011).

Los muestreos se realizaron con frecuencia semanal, seccionando la hoja $\mathrm{N}^{\circ} 25$ de dos plantas.ha ${ }^{-1}$, donde se cuantificó los huevos, instares larvales y pupas de $O$. cassina, y de sus enemigos naturales.

Se realizaron observaciones de los depredadores del insecto, así como también, de las especies vegetales que atrajeron a los potenciales enemigos naturales. El material biológico muestreado fue trasladado al laboratorio de Entomología del Instituto Nacional de Investigaciones Agropecuarias-Monagas para la obtención de adultos y de las especies de parasitoides asociados con $O$. cassina en sus diferentes fases de desarrollo.

La identificación de las especies vegetales se realizó utilizando claves, descripciones e ilustraciones disponibles en la literatura y fueron verificadas por comparación con el material disponible en los herbarios IRBR, MY, UOJ y VEN; mientras que la identificación de los depredadores se llevó a cabo a través de claves taxonómicas específicas de acuerdo a la familia del insecto.

El análisis estadístico se realizó con el programa InfoStat Versión 2018 (Di Rienzo et al., 2018) y los análisis de correlaciones comparados por la prueba de t a $5 \%$ de probabilidad.

\section{RESULTADOS Y DISCUSIÓN}

\section{Parasitoides de huevos}

En la figura 1A, se muestra el comportamiento del parasitismo global de huevos de Opsiphanes cassina. Los parasitoides actuaron principalmente desde finales de agosto hasta septiembre y desde finales de octubre hasta noviembre de 2015; de manera similar, hasta finales de enero 2016; fuera de estos lapsos su presencia no fue detectada. El porcentaje de parasitismo varió entre 21 y $100 \%$; este último, se obtuvo a finales de agosto (semana
35) y enero (semana 4). El porcentaje promedio de parasitismo en la fase de huevo fue de $61,64 \%$. Periodos muy secos o muy lluviosos podrían limitar la acción del parasitismo de huevos en el campo.

Se detectó la existencia de una correlación positiva y significativa por la prueba de $\mathrm{t}\left(\mathrm{r}=0,33^{*} p \leq 0,05\right)$ entre la población de huevos y la de sus parasitoides, de ello, se puede deducir que el efecto del parasitismo global depende del crecimiento del hospedero. Los himenópteros Telenomus sp. (Scelionidae), Anastatus sp. (Eupelmidae) y una especie no identificada perteneciente a la familia Eulophidae ejercieron el parasitismo global en la fase de huevo; en consecuencia, estos resultados armonizan con los obtenidos por Barriga (2016), de la misma manera con los resultados de Pastrana et al. (2019).

En relación con los parasitoides de huevos de $O$. cassina, Mexzón y Chinchilla (1996) han señalado que su parasitismo es poco conocido, posiblemente porque en los muestreos no son considerados por su tamaño; no obstante, en la literatura se mencionan como parasitoides de huevos a Telenomus sp. y Oencyrtus sp. (Encyrtidae) (Aldana et al., 2010; Barriga, 2016).

\section{Parasitoides de larvas de $\mathrm{V}$ instar}

En la figura 1B, se muestra el parasitismo ejercido por Cotesia sp. Este parasitoide se observó en los últimos seis meses del año, en dos lapsos, los comprendidos entre septiembre-diciembre de 2015 y de julio-agosto de 2016 , periodos estos lluviosos que coinciden con intervalos de abundancia de larvas del último instar.

Desde la semana 37 (primera quincena de agosto) a la semana 52 (finales de diciembre) el parasitismo fue variable, fluctuó entre 37,5 y $100 \%$, con promedio de $62,22 \%$. Posteriormente, durante la época seca, cuando disminuyen las poblaciones de su hospedero (larvas del V instar) el parasitoide no fue detectado. Esta circunstancia, coincide con la presentada por Argumero et al. (2000) y Pardey y Arango (2016) quienes señalaron que la población de parasitoides decrece en la época seca, aunque no desaparece. En este estudio, durante la semana 27 (inicio de julio) se detecta nuevamente el parasitoide hasta la semana 34 (finales de agosto); siendo que durante ese periodo el parasitismo fluctuó entre 16,67 y $100 \%$, con un promedio de $53,17 \%$.

Durante los intervalos de actuación del parasitoide, semanas 37 a 52/2015 y 27 a 34/2016 el porcentaje de parasitismo en las larvas del $\mathrm{V}$ instar siempre se mantuvo en promedio por encima del 50\%. Por el análisis de correlación de Pearson, entre las poblaciones de larvas del $\mathrm{V}$ instar y las del parasitoide Cotesia sp., se obtuvo una correlación positiva y significativa por la prueba de $\mathrm{t}(\mathrm{r}=$ $0,58 * p \leq 0,05)$ estos resultados sugieren que el parasitismo 
está asociado con la regulación de la población de $O$. cassina, tal argumento permite señalar a este parasitoide como el de mayor importancia. El hallazgo, está en concordancia con el trabajo realizado por Díaz et al. (2000) en las parcelas ubicadas en la localidad de El Zamuro, municipio Maturín del estado Monagas, donde identificaron a Cotesia sp. (Hymenoptera: Braconidae) y a Cotesia flavipes (Cani) entre los parasitoides de larvas; además, estos mismos investigadores también indican la presencia de Casinaria sp. y Barycerus dubiosus (Say) (Hymenoptera: Ichneumonidae) y Euphorocera sp. (Díptera: Tachinidae) y al depredador Alcaeorrhynchus grandis (Dallas) (Hemiptera: Pentatomidae), así como también encontraron una enfermedad virosa del tipo poliedrosis nuclear; de manera similar, en las condiciones de la Zona Suroccidental palmera de Colombia, Pastrana et al. (2019) también señala que Cotesia sp. ejerció parasitismo en larvas por el orden de $0,30 \%$.

\section{ESPAMCIENCIA}

En la agropecuaria "El Águila" el entomófago más activo de O. cassina fue Cotesia sp. de la familia Braconidae y se presentó solo sobre larvas del $\mathrm{V}$ instar. Detalles de larvas afectadas se muestran en la figura 2A y $2 \mathrm{~B}$. Díaz y Vásquez (2003), también lo han señalado afectando a esta plaga en plantaciones de palma aceitera en el estado Monagas. En relación con los braconidos, se menciona que son un grupo muy diverso de avispas parasitoides de lepidópteros y que entre los géneros más importantes se encuentra Cotesia sp., Fornicia sp., Digonogastra sp., Rhysipolis sp. y Rogas sp. (Mexzón y Chinchilla, 1996). En el mismo orden, Barriga (2016) observó al entomopatógeno Beauveria bassiana en larvas enfermas, con incidencia de 5,56\% y detectó a un miembro de la familia Reduvidae, al pentatomido Alcaeorrhynchus grandis y al reduvido Arilus sp. causando muerte en larvas del 68,42; 21,05, y 10,53\%, respectivamente, en la región de San Martin, Meta, Colombia.

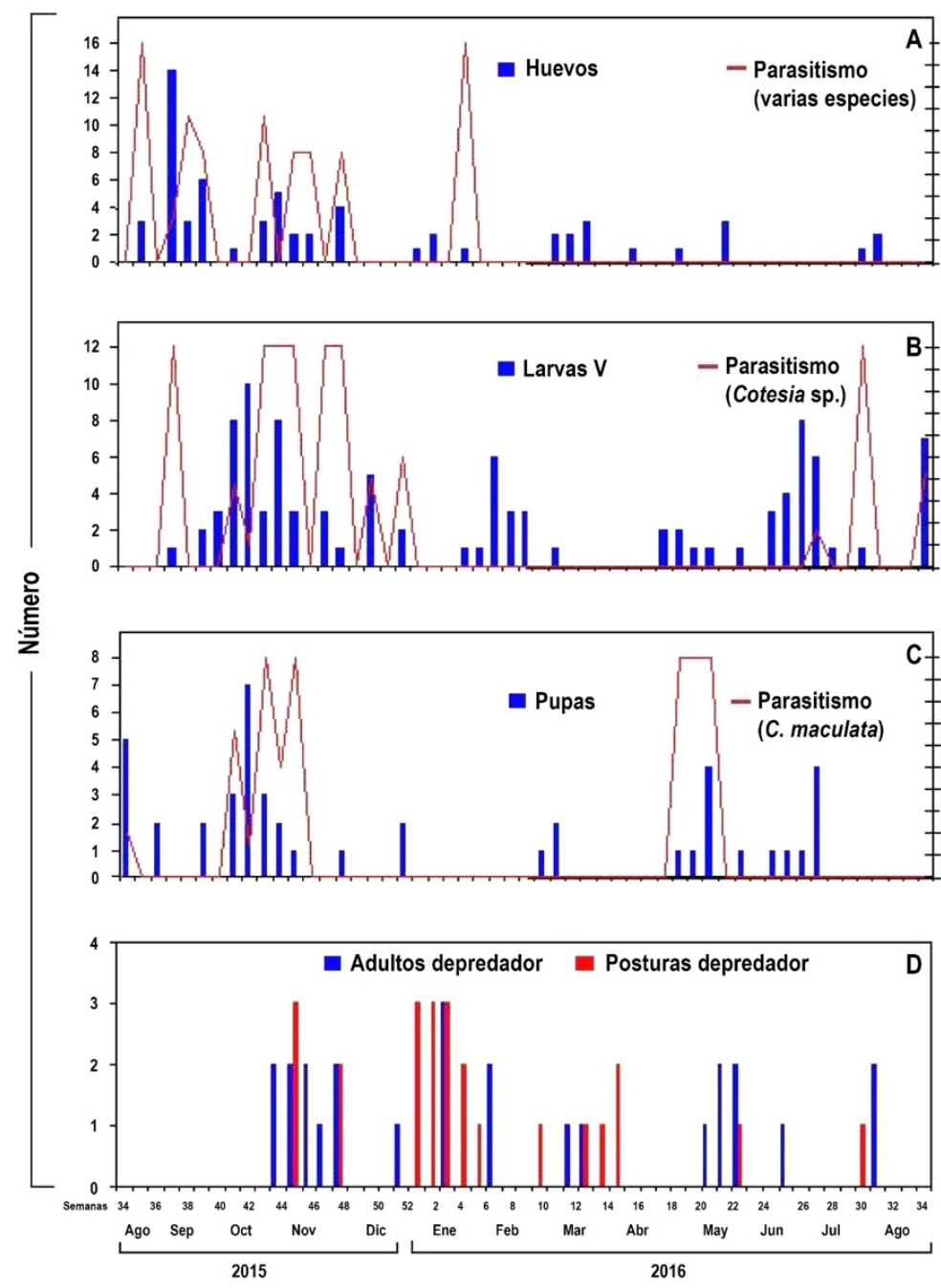

Figura 1. Parasitismo y depredación sobre Opsiphanes cassina Felder. Parasitoides en A: Huevos, por varias especies, B: Larvas V instar, por Cotesia sp, C: Pupas, por C. maculata y D: Presencia depredador, en la plantación "El Águila", Monagas, Venezuela, durante el ciclo agosto 2015 - agosto 2016. 


\section{Parasitoides de pupas}

En la figura 1C, se muestra el parasitismo de C. maculata en pupas. Este parasitoide se detectó en dos ocasiones bien definidas del año: entre los meses de octubre a noviembre (2015) y en mayo (2016). Entre la semana 41 -mediados de octubre- a la semana 45 -mediados de noviembre- el parasitismo osciló entre 14,29 y $100 \%$, con promedio de $66,19 \%$. Desapareció por un lapso bien prolongado para luego detectarse desde la semana 18 a la 20 -mayo-, en este periodo se registró parasitismo del orden de $100 \%$. Los lapsos señalados coinciden con el inicio de precipitaciones moderadas de intensidad comprendida entre 37 y $77 \mathrm{~mm}$ y de abundancia relativa de pupas. $C$. maculata, se presentó durante lapsos más corto en comparación con los parasitoides de huevos y de larvas.

La correlación de Pearson entre las poblaciones de pupas de $O$. cassina y las poblaciones del parasitoide $C$. maculata $\left(\mathrm{r}=0,37^{*} p \leq 0,05\right)$ fue positiva y significativa, por la prueba de $t$, lo que permite inferir que el parasitismo de pupa es el segundo agente de control natural importante, responsable de la mortalidad del insecto, el cual contribuye en la regulación de las poblaciones de adultos de $O$. cassina en generaciones sucesivas. Este proceso de regulación es más acentuado en las pupas existentes en la primera quincena de mayo, se observó al himenóptero $C$. maculata Fabricius de la familia Chalcididae atacando las pupas de $O$. cassina (Figura 3A, B y C), señalado también por Aldana et al. (2010); aunque, Mexzón y Chinchilla (1996) lo mencionan como parasitoide de larvas. Los adultos de este parasitoide emergen en grupos de 6 a 72 a través de un orificio circular de la pupa de su hospedero. Aldana et al. (2010), señalan que el número máximo de individuos que emergen de una pupa es de 36.

De manera similar, Díaz et al. (2000) en la localidad de el Zamuro, identificaron entre los parasitoides de pupas a Conura sp y Brachymeria sp (Hymenoptera: Chalcididae), por otro lado Barriga (2016) a nivel de pupas observo cuatro parasitoides, distinguiéndose Sarcodesia sp. (Díptera; Sarcophagidae) y Spilochalcis sp (Himenóptera: Chalcididae) responsables del 48,28 y 41,38\% respectivamente, del total de pupas parasitadas, mientras que Conura maculata, Conura sp. y Brachymeria sp. lograron en conjunto $13,44 \%$ de parasitismo en pupas (Pastrana et al., 2019), en Colombia.

\section{Parasitismo total}

De acuerdo con el análisis de correlación de Pearson se detectó una relación positiva y significativa $\left(\mathrm{r}=0,67^{*}\right.$ $p \leq 0,05)$ por la prueba de t, entre la población total de $O$. cassina (huevos, larvas $\mathrm{V}$ instar y pupas) y las poblaciones de los diversos parasitoides. Chinchilla (2003) señala que la regulación poblacional de $O$. cassina ocurre a bajas densidades por la acción de los parasitoides Telenomus sobre huevos, Conura y Cotesia sobre larvas, siendo el parasitismo en las larvas del V instar el más importante. Ese parasitismo en combinación con el de huevo y pupas, así como la captura de adultos y la acción de los depredadores contribuyen de manera significativa a la regulación de las poblaciones del insecto. Estos resultados concuerdan con lo señalado por Díaz y Vásquez (2003), quienes han considerado que la acción de los enemigos naturales de los lepidópteros defoliadores en palma aceitera, principalmente sobre $O$. cassina, desempeñan importante papel en el mantenimiento de sus poblaciones a bajos niveles. Los autores antes señalados asumen que ese impacto positivo de los agentes de control es permanente en el agroecosistema palma aceitera debido a que las relaciones ecológicas entre ellos y el ambiente son favorables.

\section{Depredadores}

Durante todo el año de evaluaciones, en la hoja $\mathrm{N}^{\circ} 25$, se encontró adultos, ninfas -las ninfas se incluyeron junto con los adultos, en una única cuantificación- y posturas de un chinche depredador no identificado perteneciente a la familia Reduviidae, al detectarse los adultos aparecen sus posturas (Figura 1D), aunque sin causar depredación a $O$. cassina. También se capturó, en una oportunidad, un ejemplar de Arilus cristatus L. (Reduviidae). Varias especies de chinches pentatómidos son mencionadas como depredadoras muy eficientes de larvas y pupas de $O$. cassina (Mexzón y Chinchilla, 1996, Chinchilla, 2003). De manera similar, Pastrana et al. (2019), señalan a los depredadores Alcaeorrhynchus grandis, Podisus sp. y tres individuos del orden Araneae ejerciendo depredación del $3,52 \%$ en larvas, pupas y adultos de $O$. cassina.

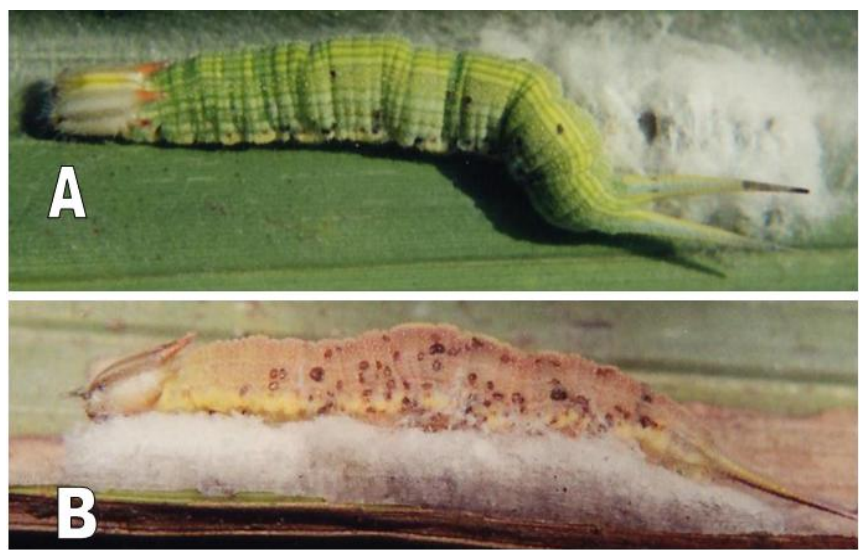

Figura 2. A y B: Larva del V instar de Opsiphanes cassina Felder parasitada por Cotesia sp. 


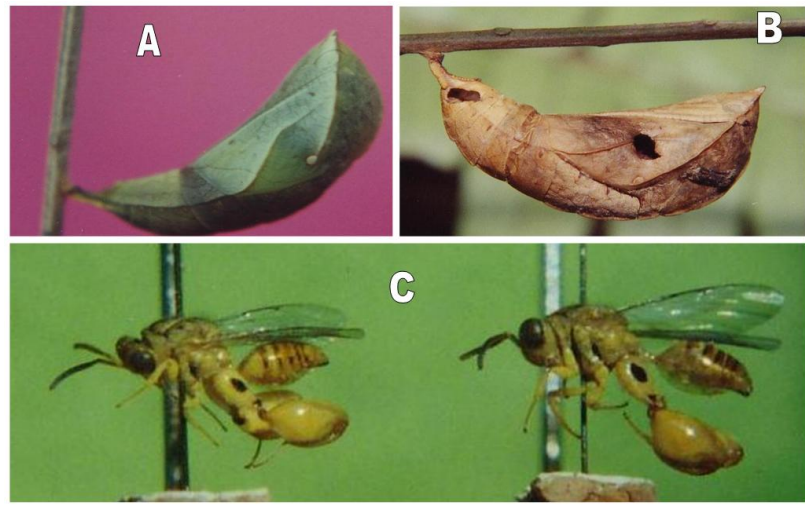

Figura 3. Parasitismo de pupa de Opsiphanes cassina Felder por Conura maculata Fabricius. (A, B): Pupas con evidencias del parasitismo y (C): Adultos de C. maculata.

\section{Vegetación benéfica}

En los cultivos de cobertura -estrato herbáceo-, varias avispitas de la familia Chalcididae fueron observadas en junio (2016) mostrando marcada preferencia, a tempranas horas de la mañana, por plantas con glándulas extraflorales, como Croton trinitatis Mills (pata de tórtola), coincidiendo con la evaluación de Díaz et al. (2000). En menor grado se observaron parasitoides en Solanum hirtum L. (huevo de gato). Posiblemente se trate de Conura maculata Fabricius, debido a que este parasitoide en mayo (2016) presentó su máximo nivel de parasitismo $(100 \%)$ en pupas, durante tres semanas consecutivas; además, su significativa actividad se detectó en junio, coincidiendo su emergencia con las pupas que fueron parasitadas. A pesar de que en esta generación, los adultos exhibieron el valor más bajo de máxima poblacional (1,3 MTD), en el campo se encontró todas las fases del insecto. En esta generación el comportamiento de los estados inmaduros tuvo tendencia similar a la primera y segunda generación, es decir, que en las primeras semanas predominó la presencia de huevos y de larvas del I, II y III instar; y en las últimas semanas larvas del IV instar, V instar y pupas. Solamente se observó parasitismo en huevos.

Barriga (2016), señaló que dentro de la vegetación asociada al cultivo, en los lotes experimentales, se encontró que Satachytarpheta cayennensis (Familia Verbenacea) atrae los adultos de los parasitoides de huevos (Telenomus sp. y un miembro de la familia Eupelmidae, otra planta atractiva, pero para los parasitoides de pupa fue Solanum hirtum (Familia Solanácea), que coinciden con los resultados obtenidos de esta investigación.

A diferencia de las generaciones descritas, la cuarta generación mostró la tendencia a incrementar las poblaciones de los primeros instares (larvas I, II y III) en la semana $20(15 / 05 / 2016)$ al final del ciclo, ya que se registró el $50 \%$ de la población de inmaduros que estuvo conformada por $30 \%$ de larvas del I instar y valor similar de $10 \%$ para los instares II y III; sin embargo, mostró similitud en cuanto a que el otro $50 \%$ correspondió al V instar $(10 \%)$ y a las pupas $(40 \%)$. En esta generación se observó parasitismo natural importante en la fase de pupa.

Al inicio (semana 21) se detectó el punto de máxima poblacional de adultos de 3,4 MTD; se encontraron huevos $(75 \%)$ y larvas III (25\%), no hubo solapamiento de generaciones, ya que no se constató la presencia de larvas de los últimos instares (IV y V), ni de pupas, debido probablemente a que aquellas fueron objeto de parasitismo al llegar a la fase de pupa. Durante la quinta generación se determinaron todas las fases del insecto, las larvas del V instar fueron afectadas por parasitoides, los cuales impidieron que muchas de ellas alcanzaran la fase de pupa. Las larvas del I instar (100\%) prevalecieron al culminar esta generación en la semana 29.

Al iniciarse la generación (semana 30), se registró huevos, larvas de los primeros (I y II) y del último instar (V), estas últimas corresponden a la generación precedente. En la semana 31 ocurrió el punto de máxima de adultos con valor de 6,1 MTD; la composición de los estados inmaduros estuvo integrada por $10 \%$ de huevos, 25 y $55 \%$ para larvas del I y II instar, respectivamente, y valor similar de 5\% para los instares III y IV. Al final del ciclo (semana 34), la población estuvo conformada por $27,27 \%$; $9,09 \%$ y $63,63 \%$ para los instares III, IV y V, respectivamente. Solamente se observó parasitismo en la población de larvas del $\mathrm{V}$ instar. En esta generación se constató huevos en presencia de adultos. No se observó la presencia de pupas durante todo el lapso, esto pudo deberse al efecto del parasitismo natural registrado en las larvas del $\mathrm{V}$ instar, lo cual no permitió que el insecto alcanzara la fase de pupa.

Los resultados expuestos indican que antes de la presencia de adultos de una nueva generación, se observan pupas en el campo. Los puntos de máximas poblacionales de adultos coinciden con la detección de huevos, que abarcan de dos o tres semanas después del pico poblacional de adultos. Conjuntamente con los huevos, pueden presentarse las larvas I, II y III o larvas V y pupas. Se infiere que las larvas del $\mathrm{V}$ instar y las pupas obtenidas en los puntos de inflexión corresponden a la generación precedente, ocurriendo solapamiento entre generaciones.

En las condiciones del estado Monagas, reviste particular importancia los cambios climáticos, especialmente los de precipitación, que pueden presentarse durante la época seca de enero a abril. Lluvias ininterrumpidas en esta época, favorecen el desarrollo de poblaciones incontrolables de larvas, ya que las plantas (sin estrés hídrico) ofrecen alimento de excelente calidad; además, el insecto, se encuentra libre de la acción de sus enemigos naturales. 
Diversos estudios hacen referencia a la necesidad del monitoreo continuo de las poblaciones de plagas, sus enemigos naturales y el favorecimiento de las plantas arvenses que le sirven de refugio en ausencia de sus hospederos (Calvache, 2004; Aldana et al., 2010; Morales et al., 2017; De Sousa et al., 2017). Por ello, es vital estar atentos y colocar trampas antes que las mariposas, inicien el periodo de oviposición.

\section{CONCLUSIONES}

El parasitismo sobre la fase de huevos, fue realizado por los himenópteros Telenomus sp. (Scelionidae), Anastatus sp. (Eupelmidae) y una especie no identificada, familia Eulophidae.

Cotesia sp., es calificado como el primer agente de control de Opsiphanes cassina, mientras que Conura maculata constituye el segundo agente de control natural, parasitando pupas de este lepidóptero-plaga.

Avispitas de la familia Chalcididae poseen preferencia por plantas con glándulas extraflorales, como Croton trinitatis; de manera similar, la presencia de parasitoides sobre Solanum hirtum, fortalecen el control biológico natural de O. cassina.

\section{AGRADECIMIENTOS}

Los autores expresan su agradecimiento al taxónomo de plantas José Gregorio Fariñas Marcano del Instituto Nacional de Investigaciones Agrícolas Monagas, y a los entomólogos Rosa Briceño del Museo Entomológico "José M. Osorio" y a Francisco Díaz; ambos, Profesores del Decanato de Agronomía, de la Universidad Centro Occidental Lisandro Alvarado, Venezuela, por realizar la identificación de las especies vegetales y los depredadores del insecto plaga, respectivamente.

\section{LITERATURA CITADA}

Aldana, R., Aldana, J., Calvache, H., Franco, P. 2010. Manual de plagas de la palma de aceite en Colombia. Sena-Cenipalma, Cuarta edición. Bogotá (Colombia). 198 p.

Aldana, R., Aldana, J. 2017. Reconocimiento y manejo de insectos defoliadores y asociados a la Pestalotiopsis. Tecnologías para la agroindustria de la palma de aceite. Bogotá D.C., Colombia. 180 p.

Argumero, A.; Aldana, J.; Calvache, H.; Celis, L. 2000. Evaluación de dos especies de plantas nectaríferas en la atracción de insectos benéficos. Palmas, 21(1):201202.
Barriga, M. J. C. 2016. Reconocimiento de enemigos naturales de Opsiphanes cassina Felder (Lepidóptera: Brassolidae) en la región de San Martin-Meta. Tesis de Ingeniero Agrónomo, Universidad Nacional de Colombia, Facultad de Agronomía, Santafé de Bogotá. $128 \mathrm{p}$.

Calvache G, H. 2004. La identificación y el control de los focos iniciales en el manejo eficiente de plagas en palma de aceite. Revista Palmas, 25 (Especial), 186193.

Calvache, H. 2016. Consideraciones sobre el manejo integrado de plagas en palma de aceite (II parte). GREPALMA (Gremial de Palmicultores de Guatemala). La Palma. Boletín Técnico. (7):1-13.

Chinchilla, C. 2003. Manejo integrado de problemas fitosanitarios en palma aceitera Elaeis guineensis en América Central. Manejo Integrado de Plagas y Agroecología (Costa Rica) 67:69-82.

Chung, C.; Sim, S.; Hon, K.; Ramli, K. 1996. Sistema de inspección y vigilancia para el manejo integrado de los gusanos comedores de follaje en palma de aceite. Palmas, 17(4):51-57.

De Sousa B., A. D., Farias, P. R. S., Dionisio, L. F. S., Tinôco, R. S., da Silva, A. G., da Silva, T. A. F. 2017. Spatial and temporal distribution of Opsiphanes invirae (Lepidoptera: Nymphalidae) in oil palm, Pará State, Brazil. Revista Brasileira de Ciências Agrárias, 12(4):464-469.

Díaz-Castañeda, C. E., Contreras-Garcia, C. V., GómezTinoco, D. A. 2020. Comparación in vitro de la capacidad invasiva de dos formulaciones a base de Beauveria bassiana como control biológico de Opsiphanes cassina. Aibi Revista de investigación, Administración e Ingeniería, 7(2):3-7.

Díaz Q, A., Barrios M, R. 2007. Investigación, desarrollo e innovación tecnológica del cultivo de la palma aceitera en Venezuela. En: Anais do Workshop LatinoAmericano de Investigación en Dendê (Palma aceitera): Alternativa para Contribuir al Desarrollo Económico y Social de la Amazonía. R. López, R. Vieira y A. Souza eds. Embrapa. 103 p.

Díaz Q, A.; González, C.; Villalba, V.; Rodríguez, G. 2000. Evaluación de insectos defoliadores y de sus enemigos naturales en plantaciones de palma de aceite (Elaeis guineensis. Jacq.) del oriente de Venezuela. Palmas, 21(1):195-200.

Díaz Q, A., Vásquez L. 2003. Parasitoides de lepidópteros defoliadores de palma aceitera (Elaeis guineensis Jacq.) en Venezuela. En: Resúmenes XVIII Congreso 
Venezolano de Entomología, Sociedad Venezolana de Entomología. Maracay, Aragua, Venezuela, 2003. p 42. (Resumen 034).

Di Rienzo, J.; Casanoves, F.; González, I.; Tablada, E.; Díaz, M.; Robledo, C.; Balzarini, M. 2018. InfoStat, FCA, Universidad Nacional de Córdoba, Argentina. Disponible en: http://www.infostat.com.ar.

Escalante O, M., Rosales, L. 2007. Insectos y hongos que afectan a Opsiphanes cassina en palma aceitera del sur del Lago de Maracaibo, estado Zulia, Venezuela. PALMAS, 28 (Especial): 339-344.

Mexzón, R., Chinchilla C. 1996. Natural enemies of harmful arthropods in oil palm (Elaeis guineensis Jacq.) in Tropical America. ASD Oil Palm Papers 13: 9-33.

Morales, L. C., Neira, A. L., Becerra, J. F. 2017. Aplicación de mejores prácticas fitosanitarias en el cultivo de palma de aceite. Fedepalma. Programa sectorial de manejo fitosanitario, Unidad de Extensión. $68 \mathrm{pp}$.

Oliveira, T. A. D., Lemos, W. P., Tinôco, R. S., Barbosa, J. C., Martins, I. C. F. 2018. Validation of the sampling methodology for Opsiphanes invirae caterpillars on oil palm plantations in the Brazilian Amazon. Ciência Rural, 48(7).

Pardey, A. E. B.; Arango, C. M. 2016. Las mejores prácticas para detener el avance de la Marchitez Letal (ML) en plantaciones de palma de aceite en Colombia. Revista Palmas, 37(4):75-90.

Pastrana S, J. L.; Matabanchoy S, J. A.; Bustillo P, A. E. 2019. Enemigos naturales de Opsiphanes cassina Felder (Lepidoptera: Nymphalidae) en la Zona Suroccidental palmera de Colombia. XV Reunión técnica nacional de Palma de aceite del 25 al 27 de septiembre, Bucaramanga, Colombia. Cenipalma.
Rodríguez G, G.; Silva-Acuña, R.; Cásares M, R.; Díaz Q, A., Barrios M, R. 2011. Metodología para muestrear las fases inmaduras del defoliador Opsiphanes cassina Felder (1862) (Lepidoptera: Nymphalidae) en palma Aceitera. Revista Científica UDO 104 Agrícola, 11 (1):104-108.

Rodríguez G, G., Silva-Acuña, R.; Cásares M, R.; Barrios M, R.; Díaz Q, A.; Fariñas M, J. 2012a. Tecnología agronómica de la palma aceitera (Elaeis guineensis Jacq,) y manejo integrado de su defoliador Opsiphanes cassina Felder (Lepidoptera: Brassolidae) en plantaciones comerciales del estado Monagas, Venezuela. Revista Científica UDO Agrícola 12 (3):584-598.

Rodríguez G, G.; Silva-Acuña, R.; Cásares M, R.; Barrios M, R.; Díaz Q, A.; Fariñas M, J. 2012b. Aspectos bioecologicos del defoliador de la palma aceitera, Opsiphanes cassina Felder (Lepidoptera: Nymphalidae). Revista Científica UDO Agrícola 12 (3):617-626.

USDA (United States Department of Agriculture). 2020. Oil Crops Outlook: June 2020. USDA, Economic Research Service, OCS-20f. 8 pp. 